\title{
CONTROL OF PCNA DEUBIQUITYLATION IN YEAST
}

Alfonso Gallego-Sánchez ${ }^{1}$, Francisco Conde ${ }^{2}$, Pedro San Segundo ${ }^{2}$ and Avelino Bueno ${ }^{1,3 *}$

${ }^{1}$ Instituto de Biología Molecular y Celular del Cáncer and ${ }^{2}$ Instituto de Microbiología-Bioquímica

${ }^{3}$ Departamento de Microbiología y Genética, Campus Miguel de Unamuno,

Universidad de Salamanca/CSIC

37007 Salamanca (Spain)

Running title: PCNA deubiquitylation.

key words: PCNA / Ubiquitin / DUB / UBP / Replication / DNA damage / checkpoint / budding yeast.

Abbreviations: ATR, ATM/Rad3-related kinase; BER, base excision repair; DUB, deubiquitinating enzyme; HU, hydroxyurea; MMS, methyl methanesulphonate; NER, nucleotide excision repair; PCNA, proliferating cell nuclear antigen; PIP, PCNA interacting protein; TLS, translesion synthesis; UBP, ubiquitin-specific protease.

*Corresponding author: abn@usal.es 


\begin{abstract}
Eukaryotes ubiquitylate the replication factor proliferating cell nuclear antigen, PCNA, to tolerate DNA damage. Although in the last few years the understanding of the evolutionarily conserved mechanism of ubiquitylation of PCNA, and its crucial role in DNA damage tolerance, has progressed impressively, little is known about the deubiquitylation of this sliding clamp in most organisms. In the present review we will discuss potential molecular mechanisms regulating PCNA deubiquitylation in yeast.
\end{abstract}

\title{
The role of PCNA ubiquitylation in DNA damage tolerance
}

The root of the problem is that many types of lesions block DNA replication forks progression during S-phase and this is intrinsically dangerous: It is thought that tolerance mechanisms exist because they prevent irreversible DNA replication fork collapse when the replisome encounters a (bulky) DNA lesion so that the restrictive nature of the active site in processive DNA polymerases impedes adequate progression of the replicative machinery.

Mechanism(s) of tolerance are essential for cells to survive exposure to genotoxic agents that damage DNA (or to resist treatments with them). In all organisms, these mechanisms ensure that DNA can be replicated even when it is damaged. This is of critical importance for cells because DNA lesions at damaged sites slows or blocks the progression of DNA replication forks increasing the risk for irreversible fork collapse. DNA lesions are circumvented by low stringency DNA polymerases that are able to bypass damaged sites in a so-called translesion DNA synthesis reaction. These DNA polymerases are called translesion synthesis, TLS, polymerases. TLS polymerases are not only damage tolerant enzymes but also mutagenic because they induce an error-prone process that causes damaged-dependent mutations. Not surprisingly, in mammals mutation of TLS polymerases may be associated to genomic instability and cancer. It is well established that in eukaryotes covalent modifications of proliferating cell nuclear antigen (PCNA) by ubiquitin regulate the choice of alternative pathways to bypass DNA lesions during S-phase [1-5].

The current understanding of the field is that cells ubiquitylates PCNA to allow the change from a processive or replicative DNA polymerase for another (TLS) polymerase to, then, replicate over-processive polymerase blocking lesions in DNA. This implies that ubiquitylated PCNA signals damaged DNA. Experimental evidence suggest that the process of translesion synthesis requires the switch between polymerases in a step-wise dependent manner: High-fidelity replicative polymerases are first blocked when they encounter a given DNA lesion, then, likely as a consequence of the first step, PCNA is ubiquitylated and, third, the stalled replicative polymerase is replaced by a TLS polymerase. In contrast with replicative enzymes, TLS polymerases are low-fidelity DNA polymerases, non-processive enzymes that lack any proofreading activity and are capable of replicating over DNA lesions. Importantly, TLSpolymerases DNA lesion bypass is independent from lesion repair (the DNA damage is left 
behind, once the replisome has passed the lesion, for a later repair independent from Sphase/DNA replication).

Why the need for PCNA deubiquitylation? It is a likely consequence of the high-affinity mechanism underlying translesion DNA synthesis. Monoubiquitylated PCNA not only signals damaged DNA but it is also a powerful/specific binding site for TLS polymerases, consequently cells need to deubiquitylate PCNA to prevent these non-processive, low-fidelity DNA polymerases to sample DNA more frequently than strictly required. In fact, TLS polymerases may be constantly sampling chromatin during DNA replication, both in the presence or in the absence of ubiquitylated PCNA. However, monoubiquitylated PCNA may prolong (in time) this interaction [6].

Ubiquitylation of PCNA is induced by chemicals causing disruptive covalent modifications of DNA blocking replication and involving the accumulation of single stranded DNA. In the yeast Saccharomyces cerevisiae, PCNA is ubiquitylated during S-phase in response to detection of DNA lesions caused by methyl methanesulfonate (MMS), hydroxyurea (HU), 4-Nitroquinoline 1oxide (4-NQO), UV-light, hydrogen peroxide $\left(\mathrm{H}_{2} \mathrm{O}_{2}\right)$ and ionizing radiation [7]. In fact, it has been shown that PCNA is ubiquitylated in response to MMS and UV-light in all eukaryotic organisms studied to date (reviewed in [3]). In particular, and in response to DNA damage, PCNA is ubiquitylated in budding and fission yeast [8-10] Xenopus [11-13] and human cell lines [14,15]. Evidence also suggest that abasic lesions on DNA induce PCNA ubiquitylation because TLS DNA polymerases are required for proficient replication through abasic DNA lesions in yeast and mammalian cells $[16,17]$.

\section{Control of PCNA ubiquitylation in S.cerevisiae}

The key regulatory role of covalent modifications of sliding clamp PCNA in the control of tolerance to DNA damage is now a solidly established model in S.cerevisiae likely conserved in all eukaryotes $[2-5,18]$. Interestingly, the PCNA homotrimer can be SUMOylated or ubiquitylated at the same lysine residue (Lys164). Addition of the SUMO residue to Lys164 is controlled in yeast by the SUMO ligase complex Ubc9-Siz1 (E2-E3). PCNA is monoubiquitinated at the same Lys164 residue by the E2-E3 complex Rad6-Rad18, thus, a PCNA monomer can either be SUMOylated or ubiquitinated (at Lys164). Monoubiquitinated PCNA can be further polyubiquitinated by the E2-E3 complex Mms2-Ubc13-Rad5 whose activity is modulated by the complex Esc4-SIx4. Thus, with key regulatory implications, ubiquitylation of PCNA unavoidably occurs in a sequential manner. PCNA is first monoubiquitylated to enhance the affinity of Rev1Rev3-Rev7 error-prone DNA polymerases that facilitate translesion synthesis (TLS) and then eventually undergoes polyubiquitylation to promote template switching, the error-free component of lesion bypass that involves sister-strand recombination ([2] and references there in). SUMOylated PCNA at Lys164 prevent homologous recombination during S-phase in the budding yeats [19]. Finally, also in budding yeast, Lys127 of the sliding clamp monomer can be SUMOylated to inhibit sister chromatid cohesion by preventing Eco1 acetyltransferase binding 
to PCNA $[5,20]$.

\section{Any contribution of the ATR-checkpoint pathway to the regulation of PCNA ubiquitylation?}

A checkpoint response ensure cell viability in the face of DNA damage. This surveillance mechanism delays, or even arrest, cell cycle progression, thus, providing additional time for cells to repair damaged DNA [21-23]. It is initiated by the kinase ATR that activates a signal transduction cascade that regulate repair responses including transcription of the DNA damage response genes, activation of DNA repair processes and recruitment of proteins to sites of DNA damage. All major components, as well as major details in the regulation of the transduction pathway, are conserved in eukaryotes. In S.cerevisiae the ATR-homologue Mec1 plays a key role in the signalling cascade by phosphorylating downstream effector kinases Rad53 or Chk1 in response to lesions in DNA and to defects at DNA replication forks $[24,25]$. Studies in S.cerevisiae and S.pombe on a potential mutual dependence of the ATR checkpoint signalling and DNA damage tolerance mechanisms indicate that they are different responses to DNA damage $[7,10]$, suggesting that the two pathways evolved independently. However, in Xenopus and human cells the situation is more controversial and a regulatory role of the ATR-mediated checkpoint response or some of its components to the ubiquitylation of PCNA cannot be excluded [12,13,26-29].

\section{Ubiquitin-specific proteases in budding yeast}

In S.cerevisiae, the E2-E3 ubiquitin ligase complex that ubiquitylates PCNA in response to DNA damage during S-phase, as mentioned earlier, is well characterized (reviewed in [2]). PCNA is ubiquitylated by the Rad6/Rad18 complex during S-phase and, then, it is actively deubiquitylated. However, the nature of the yeast enzyme (or enzymes) that deubiquitylates PCNA remains unknown. In budding yeast there are 17 genes that codify for different ubiquitinspecifc proteases (therefore potential candidates), some of them have been extensively studied while the function of others remains uncharacterized (and so they are putative deubiquitinating enzymes) [30,31]. These are named UBPs (from UBP1 to UBP17), where UBP stands for ubiquitin protease. Among the characterised UBPs, the ubiquitin protease complex Bre5/Ubp3 has been circumstancially related to the cellular response to phleomycin-mediated DNA damage [32] perhaps a consequence of its role on RNA polymerase II deubiquitylation [31]. Ubp10/Dot4, a nuclear DUB, has been shown to regulate histone H2B deubiquitylation helping to localise histone deacetylase Sir2 to the telomeres [33]. Interestingly, Ubp9, a cytoplasmic DUB, and Ubp10 have been identified as in vitro checkpoint kinase Rad53 substrates [34] suggesting a potential regulatory framework in response to DNA damage. The other nuclear DUB, Ubp8 is a component of the SAGA complex and plays a role in gene activation also through deubiquitylation of histone H2B [35]. Despite these evidences the S.cerevisiae PCNA ubiquitin protease remains elusive, in part because neither genetic nor biochemical screening has been designed yet to identify potential candidates. However, a comparative analysis with characterized PCNA interacting protein, PIP, domains may reveal key information. 


\section{PIP interacting domains in yeast (S.cerevisiae) UBPs}

Assuming that PCNA deubiquitylating enzyme must interact in vivo with the sliding clamp, what sequence analysis of yeast Ubp proteins reveals is that there are no full canonical PIP domains conserved in S. cerevisiae UBPs. QxxL/I/MxxFF is a full consensus PCNA-binding site present in many proteins that interact with the processivity factor during DNA replication [36]. S.cerevisiae UBPs lack such a consensus binding site. However, direct or indirect interaction between PCNA and an Ubp regulating deubiquitylation of covalently modified PCNA must exist, because active PCNA deubiquitylation occurs as cells exit from DNA damage. Alternatively, it has been described a PIP box variant that allows Eco1 to interact with PCNA. Eco1/Ctf7 is directly coupled to PCNA to trigger chromatid cohesion during replication via a conserved PIP box variant (QxxL/l) within the N-terminal PCNA binding domain [20]. A simple analysis of the primary amino acid sequences revealed that such PIP plain variant is present a number of times in four out of seventeen UBPs in yeast, namely Ubp3, Doa4/Ubp4, Ubp8 and Dot4/Ubp10 (Figure 1).

\section{Any (auto)regulatory control on PCNA deubiquitylase(s) in yeast?}

In human cell lines, USP1 deubiquitylates PCNA constantly in the absence of DNA damage. USP1 has been identified as the DUB that deubiquitylates PCNA [37]. Upon UV-light induced DNA damage, USP1 is (auto)degraded so that PCNA becomes ubiquitylated $[37,38]$. PCNA ubiquitylation is required for mammalian cell survival after UV-irradiation, hydroxyurea and methyl methanesulfonate [39]. However, the persistence of ubiquitylated PCNA based on USP1 disapareance upon UV-irradiation is not observed when DNA replication forks progression is blocked with HU [40]. USP1 is also involved in the deubiquitylation of the Fanconi Anemia protein FANCD2 that, indeed, has a role in repair of DNA crosslinks [41,42]. Of particular interest is that inactivation of murine USP1 results in genomic instability and a Fanconi anemia phenotype [42]. An autocatalytic cleavage event in human USP1 in response to UV-irradiation suggest a mechanism of self control that eventually would allow net ubiquitylation of PCNA (as long as the DNA damage response is active). However, yeast DUBs lack the autoproteolytic domain observed in human and conserved in vertebrates USP1-like deubiquitylating enzymes [37]. Thus, yeast UBPs are unlikely to be regulated in a similar manner.

\section{Is there any mutagenesis safeguard mechanism to keep TLS polymerases in check?}

Mutation of PCNA ubiquitin protease(s) should progressively result in increasing amounts of PCNA ubiquitylation. This is potentially damaging for the cell as an increase in monoubiquitylated PCNA would allow TLS polymerases to sample DNA more frequently than required, resulting in a net increase in mutation rates. If this is the case, it should be predicted that abrogation of PCNA DUB should result in a TLS polymerases-dependent mutator phenotype. Another direct effect of the net increase in the amounts on monoubiquitylated PCNA is that mutation of yeast PCNA ubiquitin protease(s) should break the relative balance in wild- 
type cells between TLS and strand swichting bypass of DNA lesions. The Rad6/Rad18 ubiquitylation pathway is naturally unbalance towards the error-free branch, since it is estimated that strand swichting accounts for more than $70 \%$ of the lesion bypassing events [43].

In undamaged human cells, deubiquitylation of PCNA by USP1 reveals the possibility of a mechanism against the mutagenic effect of damage-tolerant DNA polymerases [44]. As mentioned before, in cycling cell lines, it has been suggested that USP1 continuously deubiquitylates the DNA replication processivity factor, PCNA, as a safeguard against errorprone translesion synthesis of DNA. When UV-light mediated DNA damage is sensed, USP1 is down-regulated by an autocatalityc-induced two-step proteolysis process. Proteolysis of USP1 allows accumulation of monoubiquitylated PCNA [37].

The deubiquitylation of PCNA may be envisioned as a control process that provides a mechanism for the cell to counterbalance the potential deleterious effect of TLS polymerases. If this is the case, it should be predicted-or so our thinking goes-that mutation of the UBP enzyme controlling PCNA deubiquitylation shoud have an impact in the balance of the bypass of a given lesion by the error-free or the error-prone branches of covalent modifications of PCNA.

\section{Model(s) for deubiquitylation of PCNA in budding yeast: Two working hypothesis}

We hypothesize simple alternative models that explain how replicative complex switches back and forth to processive DNA polymerases when a given lesion on DNA is bypassed by using TLS polymerases. We propose two different scenarios. In a first model (Figure 2) a yet uncharacterized UbpX deubiquitylating enzyme (a single DUB or a number of them) forms part of the replicative complex (thanks to its capacity to interact with PCNA). When a lesion is detected, PCNA is monoubiquitylated to regulate the interaction with TLS (error-prone) polymerases that will result in lesion bypass or with Mms2/Ubc13/Rad5 ubiquitin ligase complex with a similar, but error-free, purpose. Once the lesion is left behind, UbpX-dependent PCNA deubiquitylation will take place, thus, preventing excessive action of these non-processive, lowfidelity DNA polymerases (TLS-polymerases) or less-processive template switching, thus, allowing the return to normal replicative DNA polymerases. In the second model, ubiquitylated PCNA will signal where the DNA lesion localizes. The mechanistic details of how the DNA lesion is bypassed will not differ from the first model, however, there will be no switch back to processive DNA polymerases and mono and polyubiquitylated PCNA will help to attract also repair enzymes for a later repair at the end of the replicative phase or even, perhaps, independently from it. A major difference between these alternative models is WHEN lesion bypass occurs. In the first model lesion bypass occurs immediately after the replisome finds the DNA lesion, in the second model it may occur later during S-phase (even in G2) after 99\% of the genome has been replicated. In fact, in mammals, two lines of evidence support the second model, first, ubiquitylated PCNA remains bound to chromatin well after the lesion has been removed [28], and, second, Rev1 TLS polymerase is highly expressed at the G2/M transition 
[45]. Several predictions may be inferred from these hypothesis, in particular the second model leads to the conclusion that DNA replication will end nearby the PCNA ubiquitylation site (that is to say where the DNA lesion is). Another prediction is that deubiquitylation of PCNA will take place late (or very late) during an extended S-phase. Future studies are required to address these issues.

\section{Yeast as a model for understanding the control of PCNA deubiquitylation}

Regulation of PCNA deubiquitylation may differ significantly from yeast to mammals, and even among yeast models as it has been shown that PCNA is ubiquitylated during unperturbed DNA replication in the fission yeast S.pombe [10] while at least in some strain backgrounds of S.cerevisiae is not and PCNA only becomes ubiquitylated upon DNA damage [7]. Thus, S.cerevisiae is unlikely the model that harbours a general/eukaryotic mode of control of PCNA deubiquitylation given the data known from human cells regarding the control of PCNA deubiquitylation. However, S.pombe may control deubiquitylation of DNA replication processivity factor, PCNA, in a similar manner to multicellular eukaryotes, not only because PCNA appears to be ubiquitylated during S-phase but also because PCNA ubiquitylation further increase upon MMS-induced DNA damage. This plausible scenario does not exclude the possibility that deubiquitylation of PCNA may be controlled by an evolutionary conserved DUB, that, intriguingly, remains to be identified in budding and fission yeast.

In Escherichia coli it has been shown that TLS polymerases Pol II and Pol IV freely exchange with the replicative Pol III and form alternative replisomes, even before Pol III stall at a given lesion, to slow down fork progression. And then Pol III switches back with Pol II and Pol IV to produce a processive replisome and resume rapid replication in vitro [46]. Similarly, polymerase switching in eukaryotes may result in slow DNA replication (in MMS-mediated DNA damage) as a consequence of the increase in monoubiquitylated PCNA forms (TLS would sample more frequently DNA and prolong the interaction in MMS-perturbed S-phase). What would be the purpose of slowing down replication when the replisome encounters DNA lesions in wild-type cells? Usefully this TLS polymerases-dependent replication fork progression slowdown may give the cell additional time to repair its DNA by the BER or NER.

\section{Future perspectives}

From our point of view, three open questions remain to be answered: First, the nature of the yeast PCNA deubiquitylating enzyme (or enzymes), second, when lesion bypass occurs (prior to or after encountering the covalent modification of DNA that disrupt replicative fork progression) and, third, if there is a switch that restores in vivo processive DNA replication resumption once the replisome has passed the lesion (on the understanding that the replisome contains both processive and non-processive DNA polymerases). A critical point (common to all three questions) is the analysis of the timing of PCNA deubiquitylation during the cell cycle. 


\section{Funding}

Work in our laboratories is funded by the Spanish Science Ministry, Junta de Castilla y León and Health Institute Carlos III (Spain).

\section{References}

1 Friedberg, E.C. (2005) Suffering in silence: the tolerance of DNA damage. Nat. Rev. Mol. Cell. Biol. 6, 943-953

2 Andersen, P.L., Xu, F. and Xiao, W. (2008) Eukaryotic DNA damage tolerance and translesion synthesis through covalent modifications of PCNA. Cell. Res. 18, 162-173

3 Chang, D.J. and Cimprich, K.A. (2009) DNA damage tolerance: when it's OK to make mistakes. Nat. Chem. Biol. 5, 82-90

4 Ulrich, H.D. (2009) Regulating post-translational modifications of the eukaryotic replication clamp PCNA. DNA Repair 8, 461-469

5 Bergink, S. and Jentsch, S. (2009) Principles of ubiquitin and SUMO modifications in DNA repair. Nature 458, 461-467

6 Sabbioneda, S., Gourdin, A.M., Green, C.M., Zotter, A., Giglia-Mari, G., Houtsmuller, A., Vermeulen, W. and Lehmann, A.R. (2008) Effect of proliferating cell nuclear antigen ubiquitination and chromatin structure on the dynamic properties of the Y-family DNA polymerases. Mol. Biol. Cell 19, 5193-5202

7 Davies, A.A., Huttner, D., Daigaku, Y., Chen, S. and Ulrich, H.D. (2008) Activation of ubiquitindependent DNA damage bypass is mediated by replication protein A. Mol. Cell 29, 625-636

8 Hoege, C., Pfander, B., Moldovan, G.L., Pyrowolakis, G. and Jentsch, S. (2002) RAD6dependent DNA repair is linked to modification of PCNA by ubiquitin and SUMO. Nature 419, 135-141

9 Stelter, P. and Ulrich, H.D. (2003) Control of spontaneous and damage-induced mutagenesis by SUMO and ubiquitin conjugation. Nature 425, 188-191

10 Frampton, J., Irmisch, A., Green, C.M., Neiss, A., Trickey, M., Ulrich, H.D., Furuya, K., Watts, F.Z., Carr, A.M. and Lehmann, A.R. (2006) Postreplication repair and PCNA modification in Schizosaccharomyces pombe. Mol. Biol. Cell 17, 2976-2985

11 Leach, C.A. and Michael, W.M. (2005) Ubiquitin/SUMO modification of PCNA promotes replication fork progression in Xenopus laevis egg extracts. J. Cell. Biol. 171, 947-954

12 Chang, D.J., Lupardus, P.J. and Cimprich, K.A. (2006) Monoubiquitination of proliferating cell nuclear antigen induced by stalled replication requires uncoupling of DNA polymerase and mini-chromosome maintenance helicase activities. J. Biol. Chem. 281, 32081-32088

13 Göhler, T., Muñoz, I.M., Rouse, J. and Blow, J.J. (2008) PTIP/Swift is required for efficient PCNA ubiquitination in response to DNA damage. DNA repair $7,775-787$

14 Kannouche, P.L., Wing, J. and Lehmann, A.R. (2004) Interaction of human DNA polymerase eta with monoubiquitinated PCNA: a possible mechanism for the polymerase switch in response to DNA damage. Mol. Cell 14, 491-500

15 Watanabe, K., Tateishi, S., Kawasuji, M., Tsurimoto, T., Inoue, H. and Yamaizumi, M. (2004) Rad18 guides poleta to replication stalling sites through physical interaction and PCNA monoubiquitination. EMBO J. 23, 3886-3896 
16 Avkin, S., Sevilya, Z., Toube, L., Geacintov, N., Chaney, S.G., Oren, M. and Livneh, Z. (2006) p53 and p21 Regulate Error-Prone DNA Repair to Yield a Lower Mutation Load. Mol. Cell 22, 407413

17 Pages, V., Johnson, R.E., Prakash, L. and Prakash, S. (2008) Mutational specificity and genetic control of replicative bypass of an abasic site in yeast. Proc. Nat. Acad. Sci. USA 105: 1170-1175

18 Moldovan, G.L., Pfander, B. and Jentsch, S. (2007) PCNA, the Maestro of the Replication Fork. Cell 129, 665-679

19 Pfander, B., Moldovan, G-L., Sacher, M., Hoege, C. and Jentsch, S. (2005) SUMO-modified PCNA recruits Srs2 to prevent recombination during S phase. Nature 436, 428-433

20 Moldovan, G.L., Pfander, B. and Jentsch, S. (2006) PCNA controls establishment of sister chromatid cohesion during $S$ phase. Mol. Cell 23, 723-732

21 Nyberg, K.A., Michelson, R.J., Putnam, C.W. and Weinert, T.A. (2002) Toward maintaining the genome: DNA damage and replication checkpoints. Annu. Rev. Genet. 36, 617-656

22 Bartek, J., Lukas, C. and Lukas, J. (2004) Checking on DNA damage in S phase. Nature Rev. Mol. Cell. Biol. 5, 792-804

23 Cobb, J.A., Shimada, K. and Gasser, S.M. (2004) Redundancy, insult-specific sensors and thresholds: unlocking the S-phase checkpoint response. Curr. Opin. Genet. Dev. 14, 292-300

24 Sanchez, Y., Desany, B.A., Jones, W.J., Liu, Q., Wang, B. and Elledge, S.J. (1996) Regulation of RAD53 by the ATM-like kinases MEC1 and TEL1 in yeast cell cycle checkpoint pathways. Science 271, 357-360

25 Sanchez, Y., Bachant, J., Wang, H., Hu, F., Liu, D., Tetzlaff, M. and Elledge, S.J. (1999) Control of the DNA damage checkpoint by Chk1 and Rad53 protein kinases through distinct mechanisms. Science 286, 1166-1171

$26 \mathrm{Bi}$, X., Barkley, L.R., Slater, D.M., Tateishi, S., Yamaizumi, M., Ohmori, H. and Vaziri, C. (2006) Rad18 regulates DNA polymerase kappa and is required for recovery from S-phase checkpoint-mediated arrest. Mol. Cell. Biol. 26, 3527-3540

27 Soria, G., Podhajcer, O., Prives, C. and Gottifredi, V. (2006) P21Cip1/NAF1 downregulation is required for efficient PCNA ubiquitination after UV irradiation. Oncogene 25, 2829-2838

28 Niimi, A., Brown, S., Sabbioneda, S., Kannouche, P., Scott, A., Yasui, A., Green, C. and Lehmann, A. (2008) Regulation of proliferating cell nuclear antigen ubiquitination in mammalian cells. Proc. Natl. Acad. Sci. USA 105, 16125-16130

29 Yang, X.H., Shiotani, B., Classon, M. and Zou, L. (2008) Chk1 and Claspin potentiate PCNA ubiquitination. Genes \& Dev. 22, 1147-1152

30 Wilkinson, K.D. (1997) Regulation of ubiquitin-dependent processes by deubiquitinating enzymes. FASEB J. 11, 1245-1256

31 Kvint, K., Uhler, J.P., Taschner, M.J., Sigurdsson, S., Erdjument-Bromage, H., Tempst, P. and Svejstrup, J.Q. (2008) Reversal of RNA polymerase II ubiquitylation by the ubiquitin protease Ubp3. Mol. Cell. 30, 498-506

32 Bilsland, E., Hule, M., Bell, S.D., Sunnerhagen, P. and Downs, J.A. (2007) The Bre5/Ubp3 ubiquitin protease complex from budding yeast contributes to the cellular response to DNA damage. DNA repair $6,1471-1484$

33 Tolga-Emre, N.C., Ingvarsdottir, K., Wyce, A., Wood, A., Krogan, N.J., Henry, K.W., Li, K., Marmorstein, R., Greenblatt, J.F., Shilatifard, A. and Berger, S.L. (2005) Maintenance of low 
histone ubiquitylation by Ubp10 correlates with telomere-proximal Sir2 association and gene silencing. Mol. Cell 17, 585-594

34 Ptacek, J., Devgan, G., Michaud, G., Zhu, H., Zhu, X., Fasolo, J., Guo, H., Jona, G., Breitkreutz, A., Sopko, R., McCartney, R.R., Schmidt, M.C., Rachidi, N., Lee, S-J., Mah, A.S., Meng, L., Stark, M.J.R., Stern, D.F., Virgilio, D.de, Tyers, M., Andrews, B., Gerstein, M., Schweitzer, B., Predki, P.F. and Snyder, M. (2005) Global analysis of protein phosphorylation in yeast. Nature 428, 679-684

35 Daniel, J.A., Torok, M.S., Sun, Z.W., Schieltz, D., Allis, C.D., Yates J.R. and Grant, P.A. (2004) Deubiquitination of histone $\mathrm{H} 2 \mathrm{~B}$ by a yeast acetyltransferase complex regulates transcription. J. Biol. Chem. 279, 1867-1871

36 Warbrick, E. (2000) The puzzle of PCNA's many partners. Bioessays 22, 997-1006

37 Huang, T.T., Nijman, S.M.B., Mirchandani, K.D., Galardy, P.J., Cohn, M.A., Haas, W., Gygi, S.P., Ploegh, H.L., Bernards, R. and D'Andrea, A.D. (2006) Regulation of monoubiquitinated PCNA by DUB autocleavage. Nat. Cell. Biol. 8, 339-347

38 Huang, T.T. and D'Andrea, A.D. (2006) Regulation of DNA repair by ubiquitylation. Nat. Rev. Mol. Cell. Biol. 7, 323-334

39 Kannouche, P.L. and Lehmann, A.R. (2004) Ubiquitination of PCNA and the Polymerase Switch in Human Cells. Cell Cycle 3, 1011-1013

40 Brown, S., Niimi, A. and Lehmann, A.R. (2009) Ubiquitination and deubiquitination of PCNA in response to stalling of the replication fork. Cell Cycle 8, 689-692

41 Nijman, S.M.B., Huang, T.T., Dirac, A.M.G., Brummelkamp, T.R., Kerkhoven, R.M., D'Andrea, A,D. and Bernards, R. (2005) The deubiquitinating enzyme USP1 regulates the Fanconi anemia pathway. Mol. Cell 17, 331-339

42 Kim, J.M., Parmar, K., Huang, M., Weinstock, D.M., Ruit, C.A., Kuto, J.L. and D'Andrea, A.D. (2009) Inactivation of murine USP1 results in genomic instability and a Fanconi anemia phenotype. Developmental Cell 16, 314-320

43 Zhang, $\mathrm{H}$. and Lawrence C.H. (2005) The error-free component of the RAD6/RAD18 DNA damage tolerance pathway of budding yeast employs sister-strand recombination. Proc. Natl. Acad. Sci. USA 102, 15954-15959

44 Ulrich, H.D. (2006) Deubiquitinating PCNA: a downside to DNA damage tolerance. Nat. Cell. Biol. 8, 303-305

45 Waters, L.S. and Walker, G.C. (2006) The critical mutagenic translesion DNA polymerase Rev1 is highly expressed during G2/M phase rather than S phase. Proc. Natl. Acad. Sci. USA 103, 89718976

46 Indiani, C., Langston, L.D., Yurieva, O., Goodman, M.F. and O'Donnell, M. (2009) Translesion DNA polymerases remodel the replisome and alter the speed of the replicative helicase. Proc Natl Acad Sci USA 106, 6031-6038 


\section{Figure Legends}

Figure 1. Amino acid sequence comparison of the catalytic domain of budding yeast UBPs

The core catalytic (Cys-BOX) domain is boxed, the consensus sequence indicated on top and the critical catalytic cysteine indicated by arrows. The conserved (QxxL/I) PIP box variant present in Ubp3, Doa4/Ubp4, Ubp8 and Dot4/Ubp10 is also boxed.

Figure 2. A model for UbpX function in the regulation of PCNA deubiquitylation in budding yeast.

Once the DNA lesion is bypassed, UbpX (one or more than one DUB) deubiquitylates the replication factor PCNA for cells to resume processive DNA replication. Different enzymes may deubiquitylate mono and polyubiquitylated PCNA, in the model we present one DUB (UbpX) for simplicity. 
S.cerevisiae UBPs encoded proteins at the catalytic domain

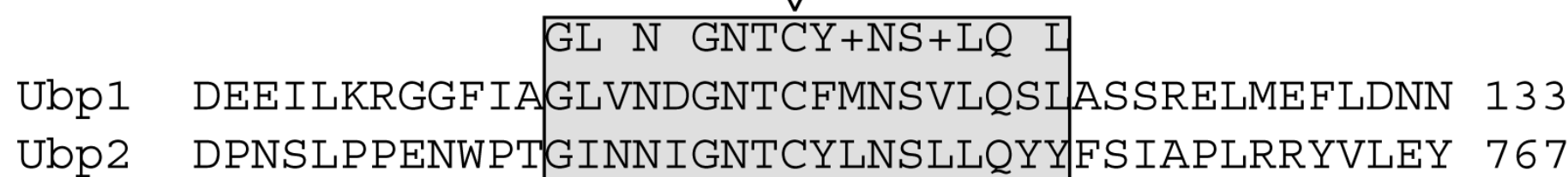
Ubp3 ENKI PVHSI IPRGI INRANICFMSSVLQVLLYCKPFIDVINVL 491 Ubp4 TSSHNYDLDFAVGLENLGNSCYMNCI IQCILGTHELTQIFLDD 593 Ubp5 NNSQVLDLDLIVGLENIGNCCYMNCILQCIVGTHDLVRMFLDN 597 Ubp6 EQQVQQFAQLPVGFKNMGNTCYLNATLQAI YRVNDLRDMILNY 140 Ubp7 RSPNVYVSLSITGLRNLGNTCYINSMIQCI FAAKTFRTLFISS 640 Ubp8 MVPSMERRDGLSGLINMGSTCFMSSILQCLIHNPYFIRHSMSQ 171 Ubp9 LMPYGDGSNKVFGYENFGNTCYCNSVLQCI YNIPEFRCNVLRY 165 Ubp10 NWGDKFTNLKPRGLLNHGVTCYTNAAVQAMLHI PS IQHYLFDI 393 Ubp11 DSNDLLSELSITGLQNPCNTCYINSIIQCIFGTTLFRDLFLTK 329 Ubp12 AYNKLEPASGTTGLVNLGNTCYMNSALQCLVHIPQLRDYFLYD 395 Ubp13 SMPYGDGSNKVFGYENFGNTCYCNSVLQCI YNLSSLRENILQF 171 Ubp14 FEKLSASKNYGCGLINLGNSCYLNSVIQSLVNGGVPNWSLDFL 376 Ubp15 LNYDSKKVTGYVGFRNQGATCYLNSLLQSYFFTKYFRKLVYEI 236 Ubp16 DSKQSIGKYTTVGLINRGNDCFITSSLQGIAGIPRFVEYLKRI 84 Ubp17 (no homology detected) 

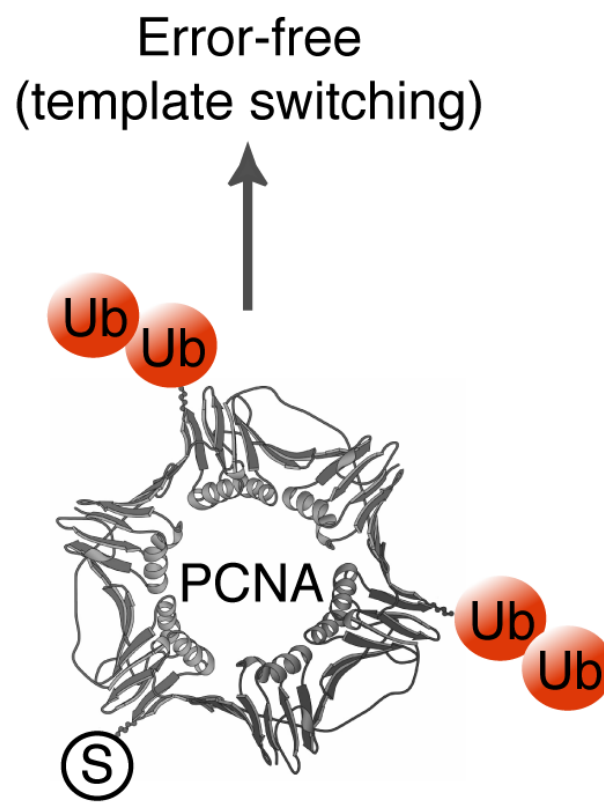

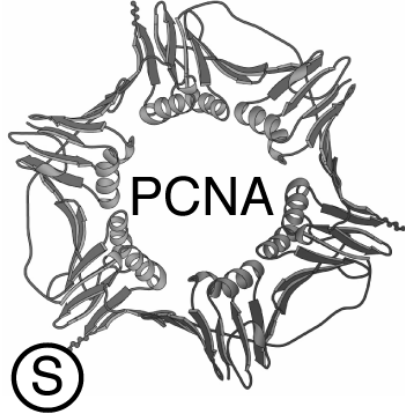

before lesion is detected
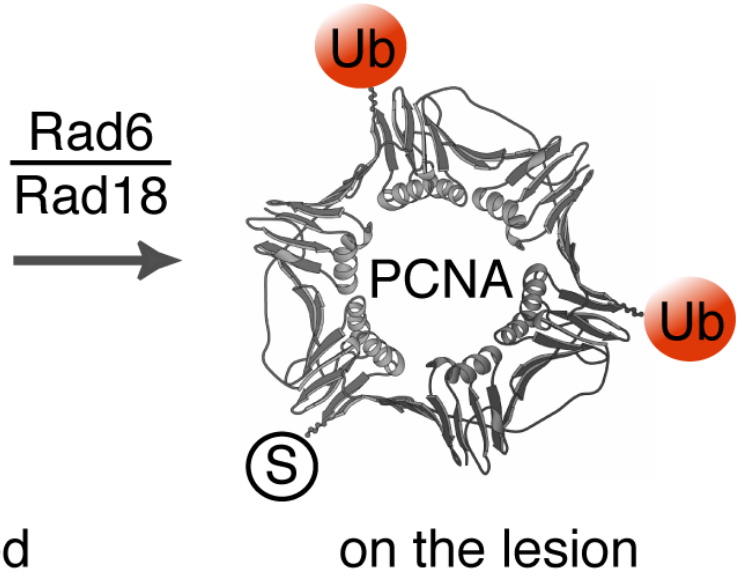

$$
\begin{gathered}
\text { Rev1,3,7 } \\
\text { Error-prone } \\
\text { (translesion synthesis) }
\end{gathered}
$$
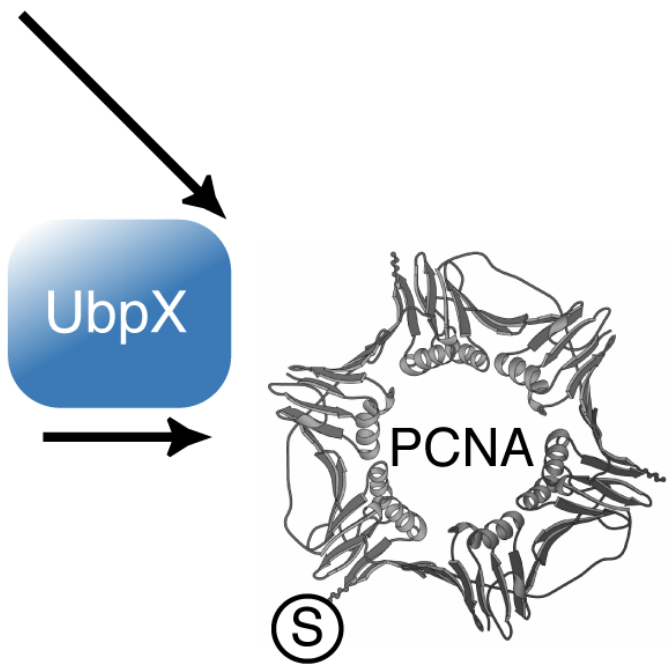

after lesion bypass 